\title{
Mathematics education and contemporary theory: guest editorial
}

\author{
Tony Brown • Margaret Walshaw
}

Published online: 17 March 2012

(C) Springer Science+Business Media B.V. 2012

All my books, are if you like, little tool boxes. If people want to open them, or to use this sentence or that idea as a screwdriver or spanner to short-circuit, discredit or smash systems of power, including eventually those from which my books have emerged, so much the better (Foucault ${ }^{1}$ ).

The inaugural Mathematics Education and Contemporary Theory conference was held in July 2011 at Manchester Metropolitan University. Primarily through discussion and the advance circulation of papers, the invitational conference with about 40 delegates asserted a place for theory in the future development of mathematics education research, with this journal special issue as an immediate target. The production of this ESM special issue comprised a key activity associated with the conference, where delegates were invited to submit papers for consideration. The papers that were finally accepted have all navigated their way through several rounds of reviews. First, authors were given the opportunity to present their papers to the conference. Second, feedback from both conference attendees and an open review process initiated revisions to papers. The revised papers were then submitted for consideration through the official journal channels for formal review.

The papers in this special issue explore the frontiers and possible futures of mathematics education research through considering how theoretical resources enable alternative possibilities. They offer a set of theoretical, narrative, empirical and practical applications of alternative concepts to and around the field of mathematics education. They do that principally by discussing and applying wider theoretical resources and demonstrating how these resources enable alternative understandings and other possibilities. To that end, the special issue contains substantial essays which make a case for theory in the future development of mathematics education research.

${ }^{1}$ Interview with Roger-Pol Droit, Le Monde, 1975.

T. Brown $(\square)$

Manchester Metropolitan University, Manchester, UK

e-mail: a.m.brown@mmu.ac.uk

M. Walshaw

Massey University, Massey, New Zealand 
The importance of considering wider theoretical resources within mathematics education is based on our belief that mathematics education, as a field for research, often relates awkwardly to its two constituent terms. These two terms - mathematics and educationinvariably wave tenuously to each other from disparate conceptual domains. Mathematics, as the first constituent term, is considered by many as a discipline beyond social discourses. Underlying this understanding is a philosophical position that seems to assert the objectivity of mathematics as a prized possession. This kind of grounding has the effect of conceptualising mathematics as constituted by pre-existing patterns that are stable and are able to be discovered. In this view, which is typically named as positivist, it is possible to know what is and what is not true since knowledge is objective and universal. In this formulation, the reality of mathematics has the same qualities regardless of observer and context.

Philosophies of mathematics which are centred in positivistic notions of mathematical truth, objectivity and stable meaning are not especially disposed to the philosophical bases of education. In particular, they do not resonate well with the linguistic turn predominant in twentieth century philosophies, privileged in all three mainstream contemporary traditions: hermeneutics, analytic philosophy and postmodernism. Truth, insofar as it is entertained, is processed through language where knowledge emerges through the operation of discursive systems. As such, knowledge houses tendencies that are not always in the business of portraying a world defined by consensual harmony in which final answers might be available. Research, in these contemporary traditions, is more in the business of generating alternative analytical filters according to diverse priorities rather than supposing that a best solution could be achieved.

The second constituent term-education-is notionally a social science susceptible to interpretive analysis. Social science brings to the fore the complexity of the social world and that particular vantage point prompts the notion that people are constantly making sense of their worlds. Realities are local, specific and constructed, and, hence, values are an integral component in the meaning systems that people generate in social action. Truth, then, is not absolute and certain but is socially and experientially based, embedded in fluid social interactions. From this particular sensibility towards knowledge construction comes the understanding that the social world can only be investigated through a systematic analysis of socially meaningful action.

However, education frequently resists conceptual immersion in the broader social sciences and the analytical resources those sciences provide since, as both idea and practice, it finds itself increasingly susceptible to external definitions and overt (and covert) regulation. Curriculum decision making is split and shared unevenly between various groups that do not necessarily see eye to eye. Their differences result in disjunctions (both real and potential) between mathematics education policy setting, curriculum implementation by teachers and the conceptualisations of mathematics education by researchers. The resolution of these conflicts has in some countries resulted in an enforced homogenisation of pedagogical practices together with demands for increased testing. Research then needs to decide which side it is on, whether it is supportive of specific political agenda or resistant to those agenda, perhaps in pursuit of more intellectual ambitions.

Restrictive conceptions of mathematics and of education, like these, mean that the composite term "mathematics education" is held in place by a variety of culturally bound assumptions. Largely circumscribed by something bigger than itself, mathematics education is constituted through a dense web of power. Traces of the determining effects of power are apparent in any mathematics education community of practice. A dilemma presents itself to those involved directly with those communities: Do we conceptualise our task in terms of initiating our students into existing knowledge? Or, might our task be seen, more radically, 
as troubling the limits of that knowledge, with a view towards keeping open the prospect of our students accessing a truth that transcends the parameters of our own teaching? In other words, is it possible for students to reach beyond the frameworks that their teachers offer to produce a new future beyond our current vision? The latter option is not to be taken lightly since it requires a major shift in thinking and practice. How do we fashion a new imaginary in which teachers forego a comprehensive understanding of what their students should be able to achieve?

Thus, a key question for mathematics education can be framed in this way: Is it possible to embrace new ontological possibilities for the learner and teacher beyond established states of representation? This specific question is at the heart of this special issue. We attempt to address it from beyond more familiar styles of theorising. Yet theory, as the object of attention, is a deliberate choice. Our intention is to open up another space for talking about the field in a way that is responsive to the diverse demands it encounters and the multiple contexts that shape its practices.

Most significantly, then, we use theory as a vehicle for new productive possibilities within mathematics education. Interventionist in its objective, the special issue uses theory to rework the epistemic responses that go hand in hand with the current image of mathematics education. It offers tools to expose the conditions that operate to make divisions between people and to investigate who has what kind of access to and what kind of engagement with mathematical knowledge. The theoretical tools allow us to understand the way in which mathematics education is managed and controlled and to determine the disciplinary set of values imposed within the field. Importantly, as a means for new productive possibilities, the special issue makes a commitment to engage in political struggle to better define mathematics education itself. At the same time, however, it acknowledges that to speak of a "better definition" is to question the very trajectory of transformative change.

The papers in the special issue are developed from an understanding of mathematics education as produced through a complex history in which teachers, learners, researchers and others involved are already inscribed, and by which their very actions, talk and symbolisations are all made to signify. Interested in both diagnosing the present and offering a political contribution to the task of reconsidering our hopes for mathematics education, the papers variously enquire into the qualities of the spaces provided for teachers, students and mathematics; temporal, spatial, formal, mechanical, political, administrative, organisational, embodied, psychological, psychoanalytic, sociological, ethnic, gendered, virtual, linguistic, empirical and of course theoretical. Negotiating through the complex history of mathematics education, they address the questions: How do we understand the parameters that generate or allow alternative conceptions of "teachers", "students" and "mathematics"? Within mathematics education research, how do "teachers", "students" and "mathematics" function methodologically as research objects?

Focused on finding answers to these questions, the papers offer resources to help us understand the increasingly complex, plural and uncertain world of mathematics education. In this kind of thinking, mathematics education is viewed, not in isolation, but as a disciplinary endeavour situated at the interface of multiple and competing structures and processes. Importantly, with efforts focused away from developing foundations and establishing authority, the papers develop a scepticism towards those principles and methods that highlight what we have come to know as certain and true.

The gateway to this new knowledge and new attitude is understood in many ways, as are the trajectories that might lead us through it. The authors demonstrate that point by illustrating their differences in terms of intellectual commitments. Irrespective of their standpoint, what the papers offer are rich analyses of lived mathematics education 
experience, not with a view of capturing reality and proclaiming causes, but of understanding the complex and changing processes by which mathematical knowledge and identities are shaped. In drawing on ideas from a wide range of thinkers, the authors offer shifts in emphases from available stories from the field, revealing how different social contexts, in which mathematics education is embedded, carve out their own boundaries. Such analyses do not seek to legislate over the constitution and nature of reality. Rather, they work at illuminating how mathematical meanings and mathematical identities are produced within particular contexts and arrangements of power.

This special issue might be read as a critical interrogation into mathematics education. It investigates aspects of practice and process typically not given attention within mathematics education. Through the analyses, it is possible to develop insights about new ways of looking at, interpreting and explaining mathematics education. Most significantly, the papers offer insights about how a new imaginary might emerge as counter-actualisations to the composite image of mathematics education. Such insights speak to the necessity of formulating a different kind of subject and a different understanding of knowledge production. We believe that the time is right for this kind of imaginary within mathematics education.

Section 1 comprises papers that directly address conceptualisations of the analytical apparatus linked to ideologically marking out the territory of mathematics education. The papers typically contrast alternative mappings of the analytical terrain and the operation of power, whilst introducing new models, such as those derived from ideological critique and post-structural approaches. Each paper seeks to disrupt more familiar analytic frames towards releasing the emancipatory potential of cross-referencing seemingly disparate approaches to reveal the internal splits that support ideological functioning.

Section 2 focuses on how notions of subjectivity can be reconfigured by rethinking the parameters through which humans identify with mathematical activity. On one hand, the papers also display an aspiration to the emancipatory productivity associated with collectivised understandings of the human subject. Yet, this aspiration can be tempered through the very operation of symbolic power formatting the actions of individuals, perhaps through the absence of space conducive to certain subjective modes or delineations of the body.

Section 3 also focuses on subjective space. The papers variously apply theory in highlighting how the affordances or opportunities of the classroom's perceptual environment differentially shape reflective work by teachers and mathematical learning by students. What is noticed and why? And how do we build awareness of such noticing? How is such awareness linked to transformative practice?

\section{Re-mapping the analytical landscape}

We begin with a paper by Alexandre Pais and Paola Valero, which further marks out the field to be explored by this special issue. Specifically, the paper outlines some ways in which the field of mathematics education research might look at itself towards refreshing its scope of interest and redefining the objects that mark out its territory. Using analytical approaches developed by the contemporary theorists, Žižek and Foucault, the paper inspects the field through critiquing the ideologies that hold the field in place. It asks the question as to what theory is in mathematics education and surveys recent work that seeks to answer this question. These concerns are addressed in three domains: what is learning, how do we improve learning and how is mathematical learning distinctive? The authors problematise the effects of these three discourses in bringing mathematics education to the political, a dimension that will be highlighted in a number of the papers to follow. 
Mary Klein similarly is involved with theoretical border-crossing. She offers a double reading of work in the field, highlighting the inadequacies of contemporary psychological theory to attend to the issue of pedagogy in inquiry classrooms. A way forward is proposed through post-structural theorising. In drawing attention to differences and possibilities between psychological and poststructuralist theory, Mary analyses data from her own published work with pre-service teachers and her earlier teaching practice. She shows how post-structural educational theory might interrupt meanings which currently, in many locations, make it difficult to effect pedagogical change. The theory is used in the paper within teacher education to build a "border pedagogy" that both acknowledges the fact that many pre-service teachers lack important mathematical knowledge and skills and, simultaneously, contributes to a more emancipatory and supportive learning space.

David Stinson and Erika Bullock also work with received ideas and their task is towards hybridising critical theory with postmodern educational theory. In exploring the parameters and possibilities of the conjoint approach, they propose that critical postmodern theory allows one to engage in emancipatory projects and, at the same time, requires one to subject those projects to scrutiny. Critical postmodern theory is not simply about emancipatory practice. It makes explicit that our "good" intentions and our desire for more equitable practices are open to critique. In that sense, the theory requires a shift in thinking and in attitude and, in particular, demands that we ask: Whose interests do our intentions and practices serve? Such a sensibility draws our attention to the necessity for self-conscious critique of our own emancipatory practices. This is, in short, a praxis of uncertainty.

Julian William's paper explores some tensions at the heart of mathematical learning, such as those between: becoming practically competent on one hand and passing tests on the other; enjoying the subject as an interesting or aesthetic experience versus studying mathematics "to get ahead in life"; mathematics as right-wrong or "black-and-white" versus mathematics being open to discussion and diverse views; learning procedures "by heart" versus understanding them via the mathematical concepts on which procedures are based, and so on. Specifically, "use" and "exchange" categories are shown to be helpful concepts in considering how mathematical education enables its students to engage with productive activity both through the productive capabilities that it confers but also through its value as an indicator of such capabilities allowing entry to privileged domains. Contemporary activity theory and Bourdieu's theory of symbolic violence provide two critical perspectives on the concept of use-value in education.

The split between use and exchange is echoed in Sverker Lundin's opposition between mathematical learning being seen as mechanical cramming, wrecking to the intellect in the service of examination performance, rather than the affordance of a powerful means to understand and control one's social and physical reality. Using psychoanalytic theory, the argument developed in Sverker's paper is that a critical stance towards mathematics education depends on an internal split of the subject of mathematics education, between the social "body" of schooling and its imaginary mathematical "soul". It describes how this idealised mathematical soul of mathematics education is made believable through its institutional organisation and demonstrates how it contributes to the distribution of students according to their level of knowledge. A central theme of the article is the perceived discrepancy between mathematics education as it is and how it supposedly could and should be in light of the properties of mathematics. The analysis implies that this gap actually is an effect of play and thus an inherent property of mathematics education itself.

Gelsa Knijnik's central focus is on ethnomathematics and on how it might be extended to include power within its basic premises. In introducing ideas from Wittgenstein and from Foucault, the paper draws attention to the need to make power explicit in the discussions that 
highlight the diversity of mathematical procedures and the relevance of culture to mathematics. Using theoretical tools such as "language games", "family resemblances", "discourse" and "resistance", she explores the politics of knowledge, providing evidence of different rationalities and different logics co-existing with western forms of school mathematics. Under discussion are the units of land measurement used in the past in Brazil and the measurement practices employed by peasants belonging to the present-day Brazilian Landless Movement. Underwriting the discussion is a concern with the subjugation of "inexact", yet imminently useful measurement calculations within agricultural practices and the coercive procedures in place that operate to maintain the circulation of western practices.

\section{Subjective spaces for teaching and learning}

Luis Radford argues that the project of modernity was built on the idea of a sovereign individual. Neatly articulated by Kant and the philosophers of the Enlightenment, this idea was transposed into education and served as the building block to shape the model of the student: a rational agent who follows a highly individualistic pattern of development and carries in itself its own projects of life. Unencumbered by communitarian and cultural concerns, this agent interacts with others moved by an individualistic logic of best selfinterest in its pursuit of knowledge. It is this concept of the psychologically defined individual that has informed most of the research in mathematics education. In his article, Luis draws on Marx, Arendt and Foucault in order to sketch what could be a culturally committed idea of mathematical knowledge, freedom and subjectivity and shows how this idea can be useful to envision non-oppressive classroom mathematics practices.

Symbolic power is discussed by Ole Skovsmose with reference to mathematics and formal languages. In general, the symbolic power of mathematics and formal languages is investigated through the following observations: (1) that mathematics treats its parts and properties as autonomous; (2) that it dismembers what it addresses and destroys the organic unit around things; (3) and that it simplifies things, designates things and reduces things to a single feature. Through these observations, Ole considers how mathematics links to conceptions of its subjects governed as they are through mathematical dimensions of their lives according to what Ole calls mechanical and formal worldviews. The idea that mathematics describes as it prioritises is discussed with reference to actions held in place through robotting and surveillance. But, whatever forms the symbolic power may take it cannot be evaluated along a single good-bad axis.

Liz de Freitas and Nathalie Sinclair conceptualise a more expansive reach for the human's expressive action. Their paper draws on the theory of Châtelet and others to explore the relationship between gesture and diagram in student problem solving. In reviewing recent mathematics education literature on the semiotic and embodied nature of gesture and diagram, a case is made for a reconceptualisation of the gesture/diagram interaction and a more fully articulated role for time and motion in mathematical thinking. Through this route, the paper challenges some of the assumptions that underlie so many instances of mathematics education research that link conceptions of the mind to particular spoken or written encapsulations of knowledge. Rather, in this paper's depiction of subjectivity, the psychological undergoes a major re-distribution towards asserting the body's temporal and spatial awareness through gesture as a radical creative act.

David Wagner discusses other difficulties relating to the complexity of human agency and subjective positioning, through examining the nature of text in pedagogical materials produced according to curriculum outcomes that demand closed tasks. The author's auto- 
ethnographic reflection is based on his school textbook writing in Bhutan in juxtaposition with his research about personal voice and positioning in mathematical texts. This work draws on film (Ellsworth) and literary theory (Eco and Barthes) with regard to reader/writer positioning in such materials. He struggles to avoid language that seductively removes the words "you" and "I" from the text, leaving only mathematics, the queen of the sciences, to be the voice of authority. The player, however, is not subject to the queen, but rather the queen is subject to the player. That is, the learner of mathematics uses the power of mathematics to exercise his or her intentions.

Yvette Solomon speculates on whether mathematics is a male domain and wonders where this leaves women who do mathematics. In a world where, Yvette argues, there is little or no discursive space in which to be female, women who enter in must do identity work in order to achieve what is often an uneasy presence. This paper builds on recent research, which suggests that some undergraduate women are however finding new spaces for belonging in the world of mathematics through critical reflection and collective challenge to dominant discourses. Focusing on an analysis of two women's narratives of their success in mathematics, it explores their multi-voiced accounts of self through the lens of Bakhtin's dialogism. It discusses the scope of reflexivity in creating new identity spaces in refigured worlds.

Margaret Walshaw and Tony Brown bring affect into the discussion of teaching and learning, drawing on contemporary readings of the work of sevententh century Dutch philosopher Baruch (Benedictus) Spinoza. In an effort to move away from formulations that draw cognition and affect by causal links, they propose that affect influences thinking just as thinking influences affect. Spinoza's work is used to demonstrate how thinking crystallises by means of the interaction between cognition and affect. In Spinoza's understanding, affect is both body and thought. Using examples drawn from two mathematics classroom sites, the paper explores the way in which affect can be conceptualised as a form of thinking that is often obscure and non-reflective, but is always already operative upon us and within us. In these examples, affect is a constitutive rather than derivative quality of classroom life.

\section{Transformative classroom practice}

Kathy Nolan's study draws on the theory of Bourdieu in exploring the theory-practice transitions experienced by secondary mathematics pre-service teachers and investigates the role these transitions play in shaping one's identity as a mathematics teacher. The paper identifies and deconstructs several (dis)positions that function as regulative discourses in secondary mathematics classrooms, working to paralyse and silence transformative possibilities in and for mathematics teaching and learning. Drawing on the theory of Bourdieu, the paper asks what a teacher educator and faculty advisor can learn from prospective secondary mathematics teachers as they construct (and are constructed by) official pedagogical discourses embedded in mathematics classrooms.

Laurinda Brown and Alf Coles are also focused on the learning environment as experienced by beginning teachers. They work with enactivist ideas to explore practices within teacher education. In introducing and drawing on the concept of deliberate analysis, they expand our thinking in relation to the practice of teacher reflection. They set up a different purpose for and a different understanding of reflection as it is typically animated in the literature. Drawing on excerpts from lessons taken from an expert teacher and the writing of a prospective teacher, their new expression of teacher reflection calls for avoidance of spontaneous action and of teacher distancing. What it does demand of the teacher is a focus 
on the relationality of the teaching/learning encounter and an engagement with uncertainty. Both these are made possible by teachers staying alert to discomfort, dissonance and the disruption of smooth functioning of their own sense making.

Victoria Hand explores the relation between teachers' professional practice and their dispositions toward equity in their teaching. She uses the notion of disposition, as grounded in the theory of a situated activity, to explain not only what teachers do and do not notice in their classroom but also to explain their moment-to-moment classroom activity. A link is forged between the influential theoretical body of work within mathematics education on noticing and with teachers' instructional practices. The argument is that learning to notice for equitable instruction shifts teachers' dispositions towards a focus on issues of culture and power in mathematics learning. Examples of equitable teaching practice are offered to highlight the ways in which teachers support learning, by means of "what" they noticed about student activity and "how" they made that noticing visible.

Melissa Gresalfi, Jacqueline Barnes and Dionne Cross explore learning opportunities made available to students. In foregrounding ideas within ecological psychology, they challenge us to think of students' engagement in terms of the system of the classroom and argue that, given the same mathematical task, specific instructional practices provide specific opportunities to learn within a more equitable classroom community. In proposing that learning is interactional, Melissa, Jacqueline and Dionne explore the instructional practices of two teachers with different personal histories but both of whom used the same instructional materials. The analysis revealed that simply providing affordances for action is insufficient on its own. Through the lens of classroom norms, Melissa, Jacqueline and Dionne showed that the dynamic intention of the teacher shapes whether and why particular affordances are acted upon by students.

Nigel Calder's paper meanwhile explores the ways in which understanding emerges when students engage with mathematical phenomena through digital technologies. Making a case for a contemporary hermeneutic perspective to meaning making, Nigel argues that mathematical learning comprises a process of interpretation, where understanding and "concepts" are states caught in ongoing formation. Working with the material of primary/elementary students' classroom discussions as they work through mathematical problems using spreadsheets and Scratch programming software, evidence is provided of how concrete practical tasks merge with hidden cognitive activity to forge new understandings. While the digital medium provides particular affordances for learning, such as the reach achieved through the management of particular classes of results, so too, do the pre-conceptions of the learners and the specific mathematical task with which students are engaged. All these-the medium, the preconceptions and the task - are interconnected and co-formative in the process of learning.

Acknowledgments The geographical locations of the guest editors often allowed $24 \mathrm{~h}$ working on this special issue, with tomorrow in New Zealand liaising with yesterday in England, at the end or beginning of a working day. Yet, this arrangement was made all the more successful by the Editor-in-Chief normally being available the same day in the USA. The guest editors wish to offer sincere thanks to Norma Presmeg for the warmly offered support and expertise provided throughout this stimulating process. 